\title{
Masgomas-4: Physical characterization of a double-core obscured cluster with a massive and very young stellar population
}

\author{
S. Ramírez Alegría ${ }^{1,2}$, A. Marín-Franch ${ }^{3}$, and A. Herrero ${ }^{4,5}$ \\ 1 The Millennium Institute of Astrophysics (MAS), Santiago, Chile \\ e-mail: sebastian.ramirez@uv.cl \\ 2 Instituto de Astrofísica, Universidad de Valparaíso, Valparaíso, Chile \\ 3 Centro de Estudios de Física del Cosmos de Aragón (CEFCA), 44001, Teruel, Spain \\ e-mail: amarin@cefca.es \\ ${ }^{4}$ Instituto de Astrofísica de Canarias, 38205 La Laguna, Tenerife, Spain \\ e-mail: ahd@iac.es \\ 5 Departamento de Astrofísica, Universidad de La Laguna, 38205 La Laguna, Tenerife, Spain
}

Received 27 October 2013 / Accepted 9 May 2014

\begin{abstract}
Context. The discovery of new, obscured massive star clusters has changed our understanding of the Milky Way star-forming activity from a passive to a very active star-forming machine. The search for these obscured clusters is strongly supported by the use of all-sky, near-IR surveys.

Aims. The main goal of the MASGOMAS project is to search for and study unknown, young, and massive star clusters in the Milky Way, using near-IR data. Here we try to determine the main physical parameters (distance, size, total mass, and age) of Masgomas-4, a new double-core obscured cluster.

Methods. Using near-IR photometry $\left(J, H\right.$, and $\left.K_{\mathrm{S}}\right)$ we selected a total of 21 stars as OB-type star candidates. Multi-object, near-IR follow-up spectroscopy allowed us to carry out the spectral classification of the OB-type candidates.

Results. Of the 21 spectroscopically observed stars, ten are classified as OB-type stars, eight as F- to early G-type dwarf stars, and three as late-type giant stars. Spectroscopically estimated distances indicate that the OB-type stars belong to the same cluster, located at a distance of $1.90_{-0.90}^{+1.28} \mathrm{kpc}$. Our spectrophotometric data confirm a very young and massive stellar population, with a clear concentration of pre-main-sequence massive candidates (Herbig $\mathrm{Ae} / \mathrm{Be}$ ) around one of the cluster cores. The presence of a surrounding H II cloud and the Herbig Ae/Be candidates indicate an upper age limit of $5 \mathrm{Myr}$.
\end{abstract}

Key words. infrared: stars - stars: early-type - stars: massive - open clusters and associations: individual: Masgomas-4 techniques: photometric - techniques: spectroscopic

\section{Introduction}

Massive stars affect the interstellar medium at several scales (by ionizing their surrounding media, depleting their native clouds through their own birth, or modifying the star formation rate in their neighbourhood), and are fundamental pieces in Galactic evolution (Martins et al. 2005). Their influence on the Galaxy takes place during their very short periods, compared with less massive stars lifetimes. We often find them deeply embedded in their natal obscured massive clusters.

Owing to high-extinction in the Galactic plane, the detection of distant Galactic massive stars and clusters using optical data becomes impossible. Near-IR photometric surveys such as 2MASS (Skrutskie et al. 2006), GLIMPSE (Benjamin et al. 2003), UKIDSS (Lawrence et al. 2007), and the ESO public survey VISTA Variables in the Vía Láctea (VVV; Minniti et al. 2010; Saito et al. 2012) have allowed the discovery of candidates in the Galaxy: for example Bica et al. (2003a,b), Dutra et al. (2003), and Froebrich et al. (2007) using 2MASS photometry; Davies et al. (2011), Kurtev et al. 2007, 2008, and Mercer et al. (2005) from GLIMPSE data; and Borissova et al. (2011) from Vista-VVV data.
Nevertheless, the census of massive clusters is far from complete; up to 100 clusters with masses greater than $10^{4} M_{\odot}$ may remain hidden (Hanson \& Popescu 2008). Systematic search programmes for these objects are necessary for a full understanding of our Galaxy. This has motivated us to develop the MASGOMAS project (Marín-Franch et al. 2009). The present phase of the project is a systematic search for massive cluster candidates in the Galactic disc. Using the preliminary version of the search algorithm we found two new massive cluster candidates in the direction of the Scutum-Centaurus arm base. This very interesting region in the Milky Way hosts a concentration of massive clusters with a red supergiant population: RSGC1 (Figer et al. 2006), RSGC2 (Davies et al. 2007), RSGC3 (Alexander et al. 2009; Clark et al. 2009), Alicante 7 (Negueruela et al. 2011), Alicante 8, (Negueruela et al. 2010), and Alicante 10 (González-Fernández \& Negueruela 2012). Our first cluster discovered in this direction, Masgomas-1 (Ramírez Alegría et al. 2012), is a spectroscopically confirmed massive cluster with an initial total mass of $(1.94 \pm 0.28) \times 10^{4} M_{\odot}$ and a coexisting population of OB-type and RSG stars.

A second cluster candidate, Masgomas- 4 , is located in the coordinates $l=40.530^{\circ}, b=+2.576^{\circ}$, or $\alpha_{2000}=18^{\mathrm{h}} 56^{\mathrm{m}} 07^{\mathrm{s}}$, 


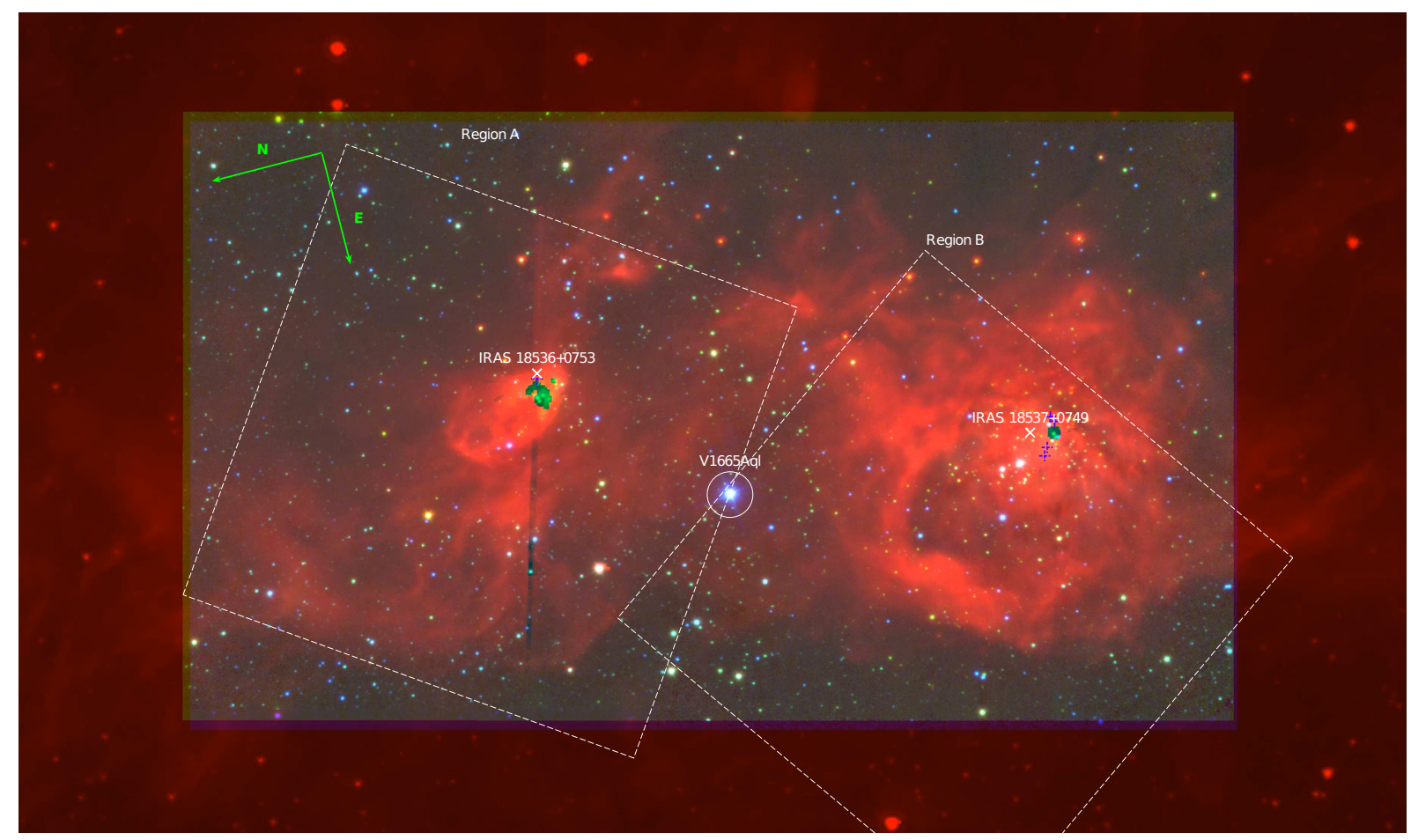

Fig. 1. Masgomas-4 false colour image $\left(J=\right.$ blue, $K_{\mathrm{S}}=$ green, $5.8 \mu \mathrm{m}=$ red). $J$ and $K_{\mathrm{S}}$ images are from LIRIS, and the $5.8 \mu \mathrm{m}$ image is from IRAC, Spitzer. The size of Spitzer image is $12^{\prime} \times 7^{\prime}$, and the size of the false colour image is $9.2^{\prime} \times 5.2^{\prime}$. White crosses show the central positions of the IRAS sources and blue crosses show the position of the masers (water, ammonia, and methanol) reported in Sect. 1. The variable object V 1665 Aql is marked with a white circle. The arrows indicate the image orientation and are 1 arcmin in length. White squares show the orientation of both multi-object masks (masks A and B).

$\delta_{2000}=+07^{\circ} 56^{\prime} 07^{\prime \prime}$. The candidate is found embedded in a bright mid-IR nebulosity, as shown in Fig. 1. Two IRAS sources are located close to Masgomas-4: IRAS 18536+0753 and IRAS $18537+0749$. Their positions are also presented in Fig. 1.

Based on these IRAS sources, we define two regions for Masgomas-4: region A (centred around IRAS 18536+0753) and region B (centred around IRAS 18537+0749). These regions have been extensively investigated in radio wavelength, but there are no studies of their stellar population ${ }^{1}$. In region A we can find methanol (Slysh et al. 1999; Szymczak et al. 2000) and hydroxyl (Baudry et al. 1997) masers, both indicative of possible massive star formation. No distance estimates are available in the literature for region A.

In region $\mathrm{B}$ we find the $\mathrm{HII}$ region Sh2-76 E (Sharpless 1959; Zinchenko et al. 1997), which can also be associated with methanol and ammonia masers. For Sh2-76 E there are two distance estimates: one of $2.1 \mathrm{kpc}$, obtained by Plume et al. (1992) using water maser observations, and a second estimate of $2.2 \mathrm{kpc}$ by Val'tts et al. (2000), using methanol maser observations ([HHG86] 185345.9+074916). The source Sh2-76 is also linked with the previously mentioned IRAS source IRAS $18537+0749$. Chan et al. $(1995,1996)$ report it as a massive young stellar object and cite two distance estimates: $2.1 \mathrm{kpc}$ (Zinchenko et al. 1994) and $2.33 \mathrm{kpc}$ (Palagi et al. 1993).

In spite of the evidence of massive star formation in both regions $\mathrm{A}$ and $\mathrm{B}$, there are no works dedicated to the observation and characterization of their stellar population. There is

\footnotetext{
An optical spectrophotometric study by Forbes (1989) included five stars from the Masgomas-4 field. Spectral classification was reported only for one B7 V star, but the author does not associate this star with the H II region.
}

only a spectral classification of B7-9 V (Ibanoğlu et al. 2007) for $\mathrm{V} 1665 \mathrm{Aql}$, an eclipsing binary located between regions A and $\mathrm{B}$. This binary system can be identified as a bright and blue source, with $K_{\mathrm{S}}=7.7 \mathrm{mag}$ and $\left(J-K_{\mathrm{S}}\right) \sim 0 \mathrm{mag}$, and according to Ibanoğlu et al. (2007), it is located at a distance of $477 \mathrm{pc}$. We will show later that this implies that the eclipsing binary is a foreground object.

Because there are no previous works dedicated to its stellar population, we cannot state whether Masgomas-4 is a single cluster or both regions A and B are two different objects. We expect to solve this question by using the individual distance and extinction estimates. In either case, we will also carry out a complete physical characterization (mass, age, star formation activity) of both regions.

In this article we describe the near-IR observations of Masgomas-4 (Sect. 2) and the near-IR photometric diagrams and spectral classifications of the 21 spectroscopically observed stars (Sect. 3). In Sect. 4 we complete an analysis of the physical parameters (distance for individual regions, cluster distance, mass, and age), and we try to determine whether Masgomas- 4 is a single cluster or two different objects. We summarize our results in Sect. 5.

\section{Observations}

We use data obtained with the LIRIS instrument (Manchado et al. 2004), a near-IR imager/spectrograph mounted at the Cassegrain focus of the $4.2 \mathrm{~m}$ William Herschel Telescope (La Palma, Canary Island, Spain). The camera is equipped with a Hawaii $1024 \times 1024 \mathrm{HgCdTe}$ array detector, with a field of view of $4.2^{\prime} \times 4.2^{\prime}$ and a spatial scale of 0 '.25 $\mathrm{pixel}^{-1}$. This work includes broadband near-IR imaging using filters 
Table 1. Summary of imaging and spectroscopic observations.

\begin{tabular}{lccccc}
\hline \hline Observing mode & Date & Filter & Exp. time & Seeing & Airmass \\
& & & {$[\mathrm{s}]$} & {$\left[{ }^{\prime \prime}\right]$} & \\
\hline Masgomas-4 field imaging & 2010 June 23 & $J$ & 108.0 & 0.78 & $1.17-1.21$ \\
$(\mathrm{~A}, \mathrm{~B}$, and control) & 2010 June 23 & $H$ & 79.2 & 0.72 & $1.22-1.27$ \\
& 2010 June 23 & $K_{\mathrm{S}}$ & 79.2 & 0.70 & $1.28-1.36$ \\
MOS $(R \sim$ 2500 $)$ & 2011 Sept. 16 & $H$ & 2520.0 & 0.83 & $1.18-1.35$ \\
Mask region A & 2011 Sept. 16 & $K$ & 1920.0 & 0.86 & $1.40-1.63$ \\
MOS $(R \sim$ 2500 $)$ & 2011 Sept. 14 & $H$ & 1800.0 & 0.78 & $1.47-1.75$ \\
Mask region B & 2011 Sept. 14 & $K$ & 2880.0 & 1.59 & $1.07-1.10$ \\
Telluric standard $(R \sim 2500)$ & 2011 Sept. 14 & $H$ & 1080.0 & 0.95 & $1.59-1.94$ \\
& 2011 Sept. 14 & $K$ & 1800.0 & 1.12 & $1.07-1.19$ \\
& 2011 Sept. 16 & $H$ & 1040.0 & 1.16 & $1.50-1.88$ \\
& 2011 Sept. 16 & $K$ & 960.0 & 1.63 & $1.94-2.56$ \\
\hline
\end{tabular}

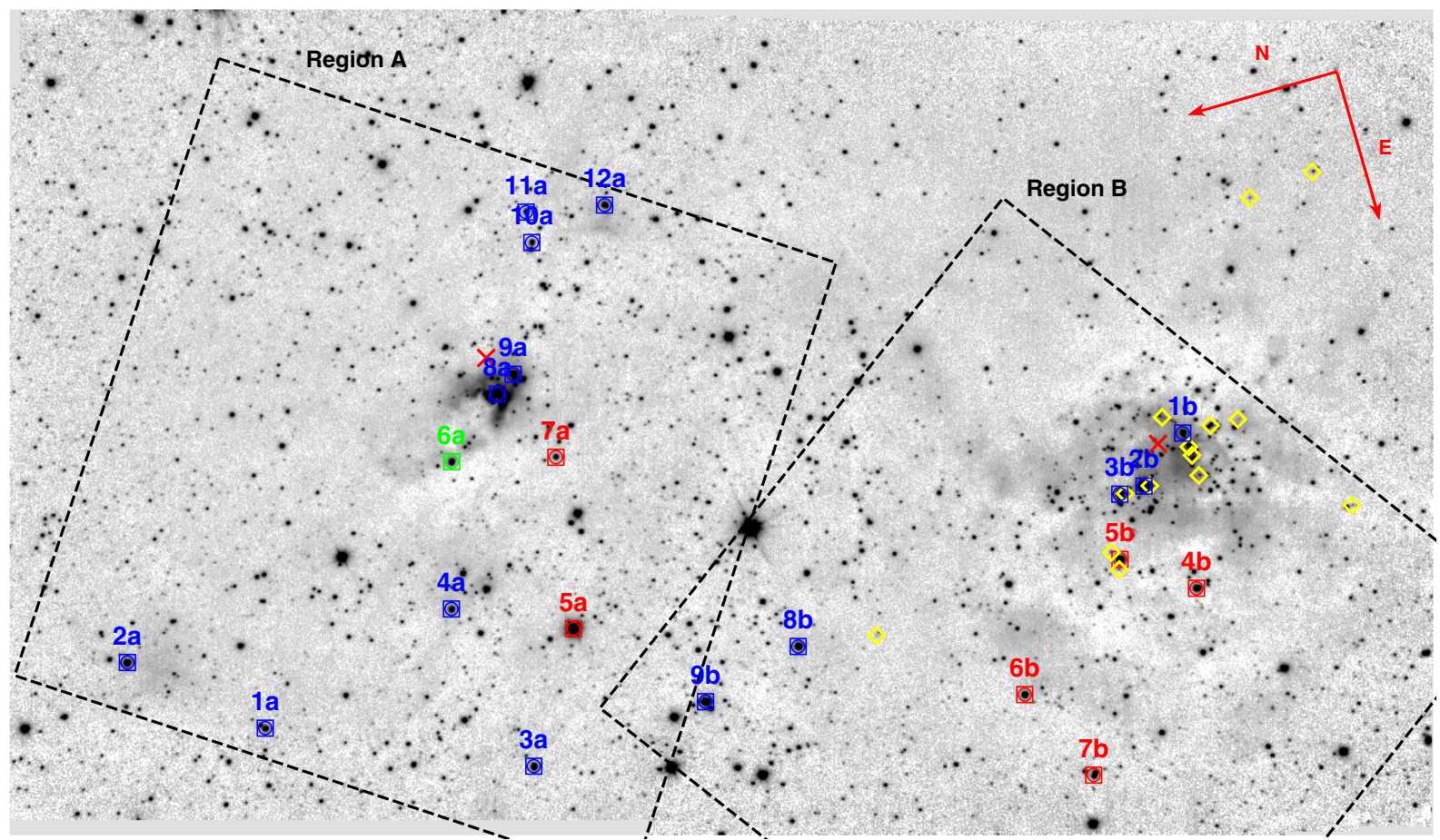

Fig. 2. Masgomas-4 $K_{\mathrm{S}}$ image obtained with LIRIS. Stars with spectral classification are marked with blue (OB-type dwarfs), green (A, F, and $\mathrm{G}$ dwarfs), and red squares (giant stars). We also mark with yellow rhombi the Herbig Ae/Be candidates and with red crosses the position of the IRAS sources. Orientation is the same as in Fig. 1 to help comparison between the figures. Length of orientation arrows is 1 arcmin, and black squares show the orientation of both multi-object masks (regions A and B).

$J\left(\lambda_{\mathrm{C}}=1.250 \mu \mathrm{m}, \Delta \lambda=0.160 \mu \mathrm{m}\right), H\left(\lambda_{\mathrm{C}}=1.635 \mu \mathrm{m}\right.$, $\Delta \lambda=0.290 \mu \mathrm{m})$, and $K_{\mathrm{S}}\left(\lambda_{\mathrm{C}}=2.150 \mu \mathrm{m}, \Delta \lambda=0.320 \mu \mathrm{m}\right)$, and multi-object mid-resolution $R \sim 2500$ near-IR spectroscopy, using pseudogrisms $H$ and $K$ (Fragoso-López et al. 2008).

The candidate apparent size is larger than the LIRIS field of view. Therefore, we divide it into two regions. Region A, centred around $\alpha_{2000}=18^{\mathrm{h}} 56^{\mathrm{m}} 05.95^{\mathrm{s}}, \delta_{2000}=+07^{\circ} 56^{\prime} 52.6^{\prime \prime}$ and including the IRAS source IRAS $18536+0753$, and region $\mathrm{B}$, centred on $\alpha_{2000}=18^{\mathrm{h}} 56^{\mathrm{m}} 11.09^{\mathrm{s}}, \delta_{2000}=+07^{\circ} 53^{\prime} 06.5^{\prime \prime}$ and containing the IRAS source IRAS $18536+0749$. The angular distance between both centres is $4^{\prime} 16^{\prime \prime}$.

We obtained the cluster and control field images on 2010 June 23 , with seeing values between $0.70^{\prime \prime}-0.80^{\prime \prime}$ (see Table 1). We observed using a nine-point dithering mode to improve cosmic-ray reduction and bad-pixel reduction, and to construct the sky image for subtraction at a later time. For image reduction (bad-pixel mask, flat correction, sky subtraction, and alignment), we followed a similar procedure to the one described by Ramírez Alegría et al. (2012). Data reduction was made with FATBOY (Eikenberry et al. 2006) and the LIRIS reduction package LIRISDR ${ }^{2}$. The final $K_{\mathrm{S}}$ image shown in Fig. 2 is a composite of regions $\mathrm{A}$ and $\mathrm{B}$ individual images.

Instrumental photometry was made with DAOPHOT II, ALLSTAR, and ALLFRAME (Stetson 1994). It was cleaned of non-stellar and poorly measured objects using the sharp index $(-0.25<$ sharp $<0.25)$ and PSF fitting $\sigma<0.1$. For photometric calibration, we used 37 stars from region A and 48 stars from region B. These stars, part of the 2 MASS catalogue (Skrutskie et al. 2006), are isolated and non-saturated stars in our photometry. For stars brighter than $J<14 \mathrm{mag}, H<11.5 \mathrm{mag}$, or $K_{\mathrm{S}}<9.5 \mathrm{mag}$, we assumed the magnitudes from the 2MASS catalogue.

For our data we define the limiting magnitude as the characteristic magnitude with a photometric error of 0.21 mag. This

2 http://www.iac.es/project/LIRIS 


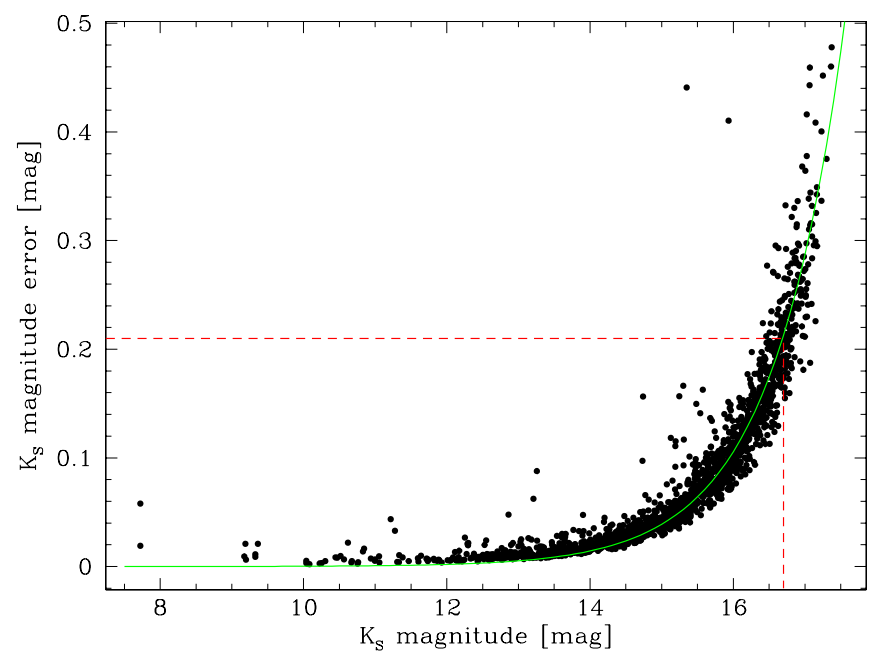

Fig. 3. Calibrated $K_{\mathrm{S}}$ magnitude versus photometric error for Masgomas-4. We show with a green solid line the exponential function fitted to the data, and with a red segmented line the limiting magnitude of $K_{\mathrm{S}} \sim 16.7 \mathrm{mag}$ associated with the photometric error of $0.21 \mathrm{mag}$ (or a signal-to-noise ratio of 5).

photometric error limit corresponds to a signal-to-noise ratio of 5 (Newberry 1991). In Fig. 3, we show the $K_{\mathrm{S}}$ magnitude and its associated error for the Masgomas-4 photometry, and the exponential function fitted to the data. Using this function we estimated that the limiting magnitude for the $K_{\mathrm{S}}$ band is $16.7 \mathrm{mag}$.

We used SKYCAT for the astrometric calibration, by correlating image and equatorial coordinates for the same set of stars used in the photometric calibration. Fitting errors are less than 0.15 arcsec for the three filters.

Near-IR spectra were observed during 2011 September 14, 15 , and 16 (half nights, Table 1). As shown in next section, two masks were designed to observe the OB-type star candidates. We included in the region A mask (hereafter mask A) twelve stars, and in the region B mask (mask B), nine stars. To avoid large signal-to-noise differences for spectra with the same exposure times, we limited the magnitude difference in each mask to less than 2 mag (for $K_{\mathrm{S}}$ ). Slits of 0.8 arcsec in width and 8-10 arcsec in length were cut on each mask, which produced a resolution of $R \sim 2500$. The typical values for the signal-to-noise is $\sim 76.7$ for $H$ band spectra, and $\sim 78.6$ for $K$ band spectra, per resolution element. We also took into account the slit position along the dispersion axis, a key factor to obtain the right wavelength range to observe OB-type characteristic spectral lines. The telluric standard for all spectra was the A0 V star HD 231033, and the modelling of the A0 V spectral contribution was carried out with XTELLCOR (Vacca et al. 2003). The subtraction of the telluric lines and the correction due to the airmass difference were completed with the IRAF task TELLURIC.

\section{Results}

In the first part of this section we show the photometric diagrams (colour-magnitude, colour-colour, and pseudocolourmagnitude) for regions $\mathrm{A}, \mathrm{B}$, and the control field. The second part of the section is dedicated to the infrared spectra from Masgomas-4, and the description of the spectral features used for the spectral classification.

\subsection{Photometry}

In the colour-magnitude diagrams (CMD) for regions A and $\mathrm{B}$ (Fig. 4) the cluster main sequence is not clearly seen. Differential extinction spread it to redder colours, following the reddening vector. This makes it difficult to distinguish the cluster stars among the field stars using only the CMD. It is therefore necessary to use extra photometric colours to clearly spot the cluster stellar population. For this purpose we used the reddeningfree parameter $Q_{\mathrm{IR}}$ (Comerón \& Pasquali 2005; Negueruela \& Schurch 2007) to identify the OB-type star candidates in regions $\mathrm{A}$ and $\mathrm{B}$.

The definition of the parameter $Q_{\mathrm{IR}}$ depends on the assumed extinction law, which can be determined from the regions' colour-colour diagrams (CCD, Fig. 5). These diagrams show that the extinction in regions $\mathrm{A}$ and $\mathrm{B}$ is accurately described by the Rieke extinction law (Rieke et al. 1989), with $R=3.09$ (Rieke \& Lebofsky 1985).

The slopes of the linear function fitted to the $\left(H-K_{\mathrm{S}}\right)-(J-H)$ diagrams are

$\frac{E(J-H)}{E\left(H-K_{\mathrm{S}}\right)}=1.69 \pm 0.03$

for region $\mathrm{A}$ and

$\frac{E(J-H)}{E\left(H-K_{\mathrm{S}}\right)}=1.66 \pm 0.04$

for region $\mathrm{B}$. These values are in agreement, within errors, with the value derived from the Rieke extinction law:

$\frac{E(J-H)}{E\left(H-K_{\mathrm{S}}\right)}=1.70$.

Using the reddening-free parameter $Q_{\mathrm{IR}}$ defined by this extinction law

$Q_{\mathrm{IR}}=(J-H)-1.7 \cdot\left(H-K_{\mathrm{S}}\right)$,

we can see in both pseudocolour-magnitude diagrams a vertical sequence around $Q_{\mathrm{IR}} \sim 0$ mag (Fig. 6). These sequences, which are understood as OB-type star candidates, are not seen in the control field's pseudocolour-magnitude diagram. Therefore, it is a feature associated with the cluster stellar population.

In the colour-magnitude diagrams we can also see a group of bright and reddened stars, close to $K_{\mathrm{S}} \sim 10 \mathrm{mag}$ and $\left(J-K_{\mathrm{S}}\right) \sim$ 4 mag. These stars could be part of the upper end of the cluster main sequence, spread by the differential extinction to dimmer magnitudes and redder colours. Because these objects have pseudocolour values of $Q_{\mathrm{IR}} \sim 0.6 \mathrm{mag}$, they are most probably giant stars from the galactic disc.

For multi-object spectroscopy we selected stars with reddening-free parameter $Q_{\mathrm{IR}}$ between $-0.2 \mathrm{mag}$ and $0.2 \mathrm{mag}$. We also selected some stars, outside this $Q_{\mathrm{IR}}$ range, due to their position in the Masgomas-4 field (see Fig. 2). For example, star 7a $\left(Q_{\mathrm{IR}}=0.50 \mathrm{mag}\right)$ is located at the centre of region $\mathrm{A}$; star $8 \mathrm{a}$ has $Q_{\mathrm{IR}}=-0.52 \mathrm{mag}$ and is also a central star in region $\mathrm{A}$; star $6 \mathrm{~b}\left(Q_{\mathrm{IR}}=0.84 \mathrm{mag}\right)$ is located on the edge of a bubble-shaped structure in region $\mathrm{B}$; star 11a has a value of $Q_{\mathrm{IR}}=0.25 \mathrm{mag}$ and, because it does not overlap with any other slit in the mask, we decided to include it.

A second group of stars are blue objects with an interesting position in the cluster field. For example star 6a, which is located in one arm-shaped structure of IRAS 18536+0753, and star $5 \mathrm{~b}$, which is close to the bubble-shaped structure of IRAS $18537+0749$. 
S. Ramírez Alegría et al.: Masgomas-4

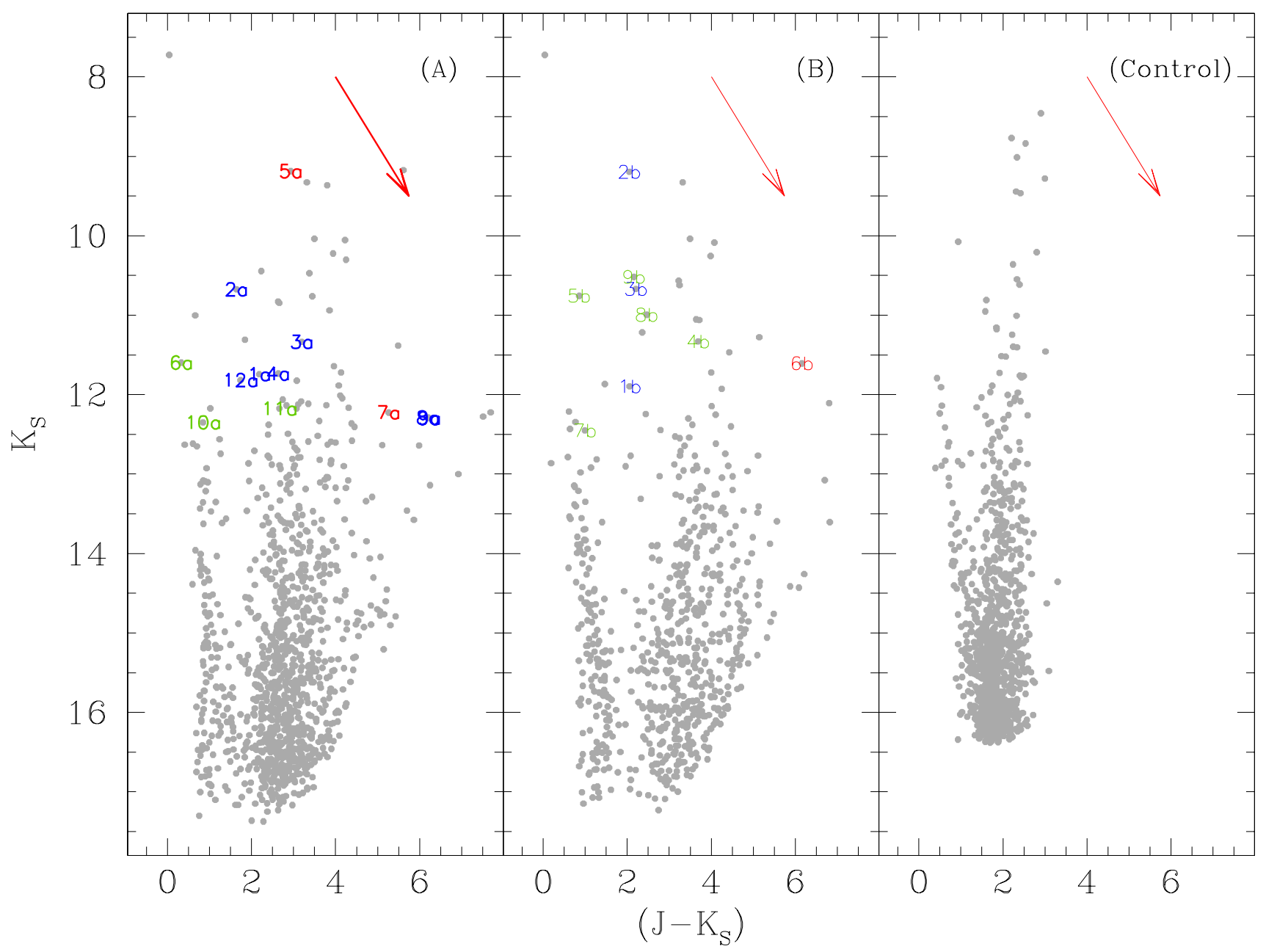

Fig. 4. Cluster colour-magnitude diagrams (CMD) for regions A (left), B (middle), and the control field (right). We label the spectroscopically observed and classified stars using their numbering for masks A and B. We indicate the OB-type stars with blue symbols; A-, F-, and G-type dwarfs with green symbols; and giant stars with red symbols. The red arrow shows an extinction of $A_{K_{\mathrm{S}}}=1.5 \mathrm{mag}$, assuming the extinction law from Rieke et al. (1989), with $R=3.09$ (Rieke \& Lebofsky 1985).

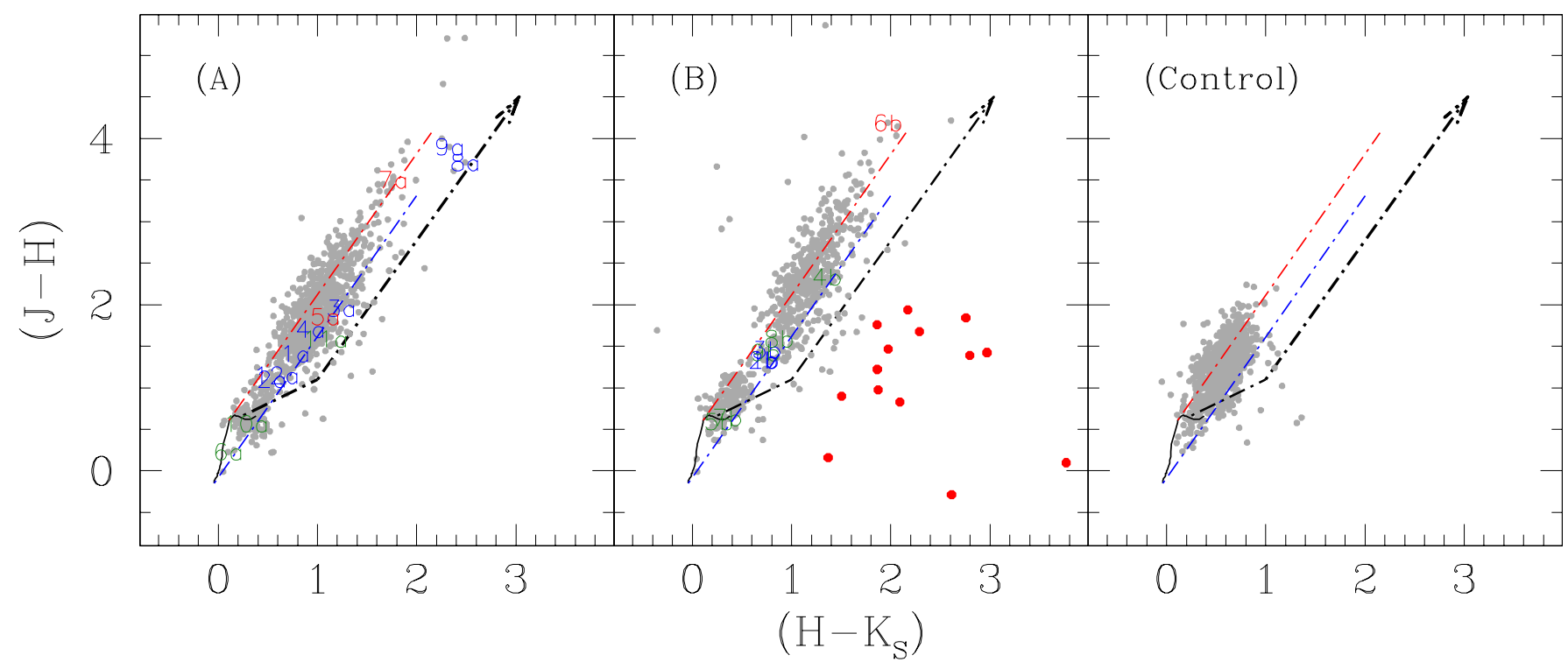

Fig. 5. Cluster CCDs for regions A (left), B (middle), and the control field (right). We use the same symbols as in Fig. 4 to mark the spectroscopically observed stars. In the diagrams we mark with segmented lines the position of an O8 V (blue lines) and a K5 V star (red lines), following the Rieke extinction law (Rieke et al. 1989), as used in this work. Thicker black segmented lines show the expected position for T Tauri stars along the extinction vector. In the region B diagram, we show with red circles the stars with infrared excess, candidates to Herbig Ae/Be objects. 


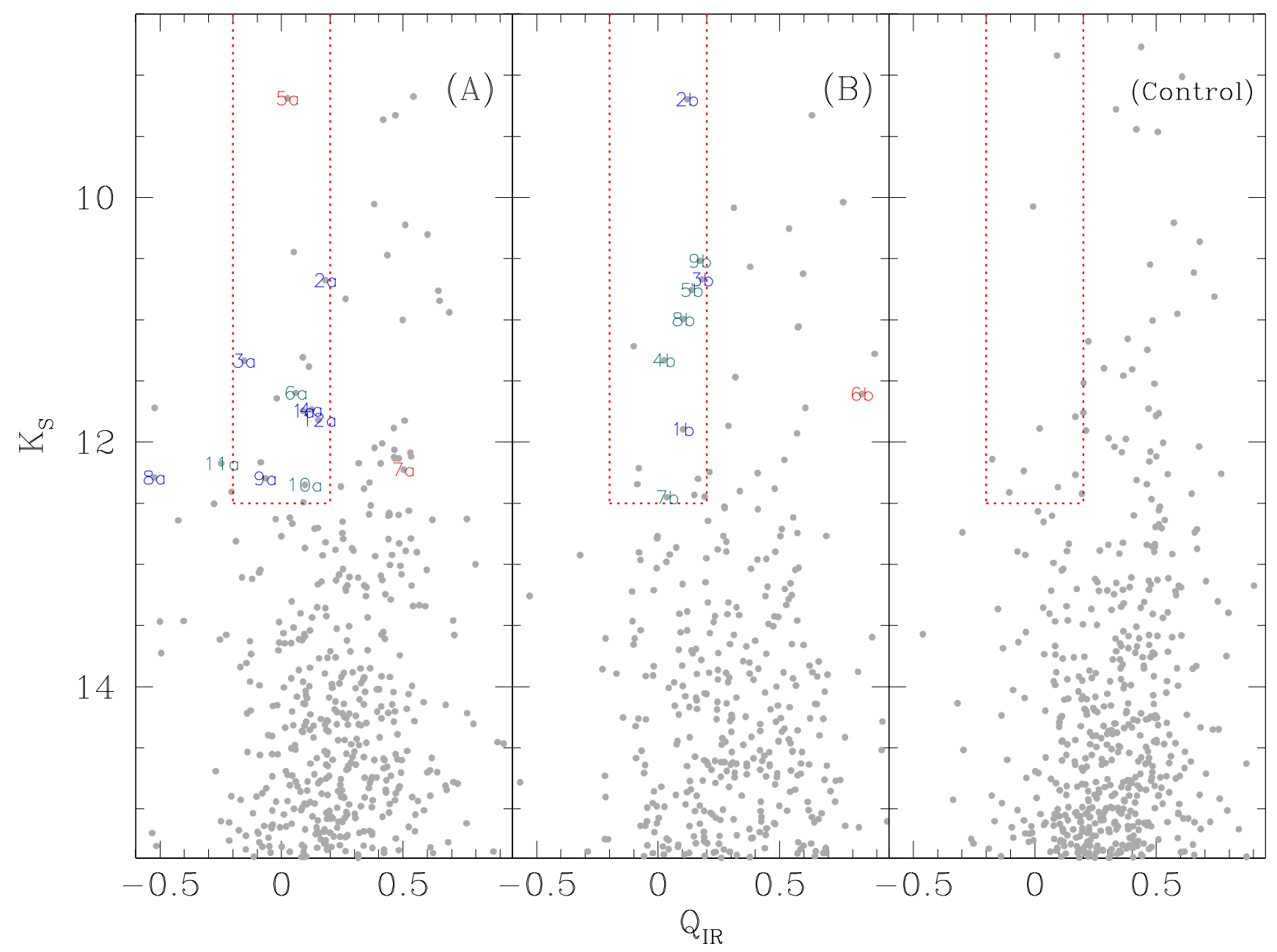

Fig. 6. Cluster pseudocolour-magnitude diagrams for regions A (left), B (middle), and the control field (right). We use the same symbols as in Fig. 4 to mark the spectroscopically observed stars (blue = OB-type, green = A-, F-, G-type, red = giants). Dotted red lines mark the adopted limits in pseudocolour $Q_{\mathrm{IR}}$ and magnitude $K_{\mathrm{S}}$ for the OB-candidates selection.

Finally we identify a group of very reddened stars in the region B CCD. This could indicate the existence of a younger (and more embedded) population in region B. These objects are shown with yellow rhombi in Fig. 2.

\subsection{Spectral classification}

Spectral classification for Masgomas-4 has followed a similar procedure to that in previous group works (G61.48+0.09, Marín-Franch et al. 2009; NGC 7538, Puga et al. 2010; Sh2-152, Ramírez Alegría et al. 2011; and Masgomas-1, Ramírez Alegría et al. 2012). The classification scheme is based on the detection of absorption lines and the comparison of their depth and shape with similar resolution spectra of known spectral types. The catalogues used for spectral classification were Hanson et al. (1996, $K$ band) and Hanson et al. (1998, $H$ band), for OB-type stars, and Meyer et al. (1998); Wallace \& Hinkle (1997) for late-type stars. For the assigned spectral type we assume an error of \pm 2 subtypes, similar to Hanson et al. (2010), Negueruela et al. (2010), and Ramírez Alegría et al. (2012).

Table 2 provides the coordinates, infrared magnitudes, and spectral types of the spectroscopically observed stars. The mask A and mask B final spectra, as well as the telluric correction spectra, are shown in Figs. 7 (OB-type stars) and 8 (AFG-type dwarfs and giants stars).
According to the stellar spectra, we can separate the observed stars in three groups: OB-type stars, AFG-type dwarfs, and giant stars.

\subsubsection{OB-type stars}

These stars are identified by their helium lines and incomplete Brackett series. According to the observed spectral features, star $2 \mathrm{~b}$ is the earliest object in our set. The spectrum has strong $\mathrm{He}$ I lines at 1.70 and $2.11 \mu \mathrm{m}$, similar to those found in $09.5 \mathrm{~V}$ stars (for example, HD 37468 or HD 149757, Hanson et al. 2005) and Brackett series similar to an O9 V star (e.g. HD 193322, Hanson et al. 1996). We have adopted a spectral type O9-9.5 V for this star.

Star $3 \mathrm{~b}$ shows features of a slightly later star. The Brackett series is deeper and similar to the B2 V HD 36166 star (Hanson et al. 2005), and its clear He I lines are similar to the B0 V HD 36512 star (Hanson et al. 1996). We classify star 3b as B1 V.

Spectra of stars 1a, 2a, and 9a show weak Brackett series, until H I (4-12), with a depth similar to a B3 V star (e.g. HR 5191, Meyer et al. 1998). The weak He I line at $1.70 \mu \mathrm{m}$ indicates a stellar type similar to a B2 V star, so we adopt a spectral type B2-3 V for these stars. The spectrum of star 1a shows an emission at $1.58 \mu \mathrm{m}$ and others stars (e.g. stars 3a, 6a, 9a, 10a, $11 \mathrm{a}$, and 12a) show an emission bump at this position. Inspecting 
Table 2. Stars with observed spectra.

\begin{tabular}{|c|c|c|c|c|c|c|c|c|}
\hline ID & $\begin{array}{c}\text { RA (J2000) } \\
{\left[\begin{array}{lll}\mathrm{h} & \mathrm{m} & \mathrm{s}\end{array}\right]}\end{array}$ & $\begin{array}{c}\operatorname{Dec}(\mathrm{J} 2000) \\
{\left[\begin{array}{lll}\circ & \prime & \end{array}\right]}\end{array}$ & $\begin{array}{c}J \\
{[\mathrm{mag}]}\end{array}$ & $\begin{array}{c}H \\
{[\mathrm{mag}]}\end{array}$ & $\begin{array}{c}K_{\mathrm{S}} \\
{[\mathrm{mag}]}\end{array}$ & Spectral type & $\begin{array}{c}A_{K} \\
{[\mathrm{mag}]}\end{array}$ & $\begin{array}{c}\text { Distance } \\
{[\mathrm{kpc}]}\end{array}$ \\
\hline \multicolumn{9}{|c|}{ OB-type dwarfs: } \\
\hline $2 b$ & 185611.98 & +07 5338.1 & $11.246 \pm 0.008$ & $9.911 \pm 0.008$ & $9.196 \pm 0.006$ & O9-9.5 V & $1.47_{-0.03}^{+0.02}$ & $1.77_{-0.67}^{+0.26}$ \\
\hline $3 b$ & 185611.99 & +075348.1 & $12.875 \pm 0.003$ & $11.419 \pm 0.004$ & $10.767 \pm 0.004$ & $\mathrm{~B} 1 \mathrm{~V}$ & $1.54_{-0.02}^{+0.03}$ & $2.08_{-0.99}^{+1.43}$ \\
\hline 1a & 185611.05 & +075931.0 & $13.926 \pm 0.014$ & $12.519 \pm 0.010$ & $11.744 \pm 0.006$ & B2-3 V & $1.52_{-0.02}^{+0.03}$ & $2.47_{-0.77}^{+2.24}$ \\
\hline $2 \mathrm{a}$ & 185608.31 & +08 0014.3 & $12.318 \pm 0.018$ & $11.217 \pm 0.002$ & $10.676 \pm 0.005$ & B2-3 V & $1.16 \pm 0.01$ & $1.52_{-0.29}^{+0.95}$ \\
\hline $9 \mathrm{a}$ & 185604.21 & +07 5717.9 & $18.526 \pm 0.014$ & $14.628 \pm 0.046$ & $12.296 \pm 0.020$ & B2-3 V & $4.19_{-0.02}^{+0.03}$ & $0.93_{-0.29}^{+0.84}$ \\
\hline $12 \mathrm{a}$ & 185600.73 & +075624.3 & $13.554 \pm 0.028$ & $12.404 \pm 0.005$ & $11.818 \pm 0.007$ & B3 V & $1.21 \pm 0.02$ & $2.13_{-0.22}^{+1.93}$ \\
\hline $1 \mathrm{~b}$ & 185610.97 & +075317.6 & $13.954 \pm 0.005$ & $12.620 \pm 0.005$ & $11.895 \pm 0.006$ & B3 V & $1.43 \pm 0.02$ & $2.00_{-0.21}^{+1.81}$ \\
\hline $4 a$ & 185609.55 & +07 5808.3 & $14.370 \pm 0.007$ & $12.665 \pm 0.007$ & $11.734 \pm 0.005$ & B3-5 V & $1.80 \pm 0.02$ & $1.48_{-0.22}^{+0.67}$ \\
\hline $3 a$ & 185614.12 & +07 5756.5 & $14.530 \pm 0.007$ & $12.575 \pm 0.004$ & $11.335 \pm 0.011$ & B0-5 V & $2.19 \pm 0.03$ & $1.68_{-0.70}^{+1.19}$ \\
\hline $8 \mathrm{a}$ & 185604.57 & +07 5726.1 & $18.489 \pm 0.092$ & $14.779 \pm 0.033$ & $12.289 \pm 0.021$ & YSO & - & - \\
\hline \multicolumn{9}{|c|}{ A, F, G dwarfs: } \\
\hline $8 b$ & 185613.22 & +075604.6 & $13.459 \pm 0.003$ & $11.847 \pm 0.003$ & $11.100 \pm 0.005$ & A5 III-V & $1.58_{-0.03}^{+0.02}$ & $0.38_{-0.03}^{+0.04}$ \\
\hline $9 \mathrm{~b}$ & 185613.86 & +075645.3 & $12.678 \pm 0.005$ & $11.254 \pm 0.005$ & $10.615 \pm 0.010$ & F3-7 V & $1.25_{-0.02}^{+0.03}$ & $0.21 \pm 0.00$ \\
\hline $6 a$ & 185605.90 & +07 5750.9 & $11.926 \pm 0.021$ & $11.697 \pm 0.004$ & $11.597 \pm 0.008$ & F6 V & $0.04_{-0.02}^{+0.03}$ & $0.61 \pm 0.01$ \\
\hline $11 \mathrm{a}$ & 185600.29 & +075653.9 & $14.839 \pm 0.009$ & $13.253 \pm 0.008$ & $12.175 \pm 0.006$ & F6-8 V & $1.56 \pm 0.02$ & $0.40 \pm 0.04$ \\
\hline $10 \mathrm{a}$ & 185601.09 & +075655.4 & $13.195 \pm 0.031$ & $12.627 \pm 0.006$ & $12.349 \pm 0.008$ & F7 V & $0.36 \pm 0.02$ & $0.74_{-0.07}^{+0.08}$ \\
\hline $5 b$ & 185613.62 & +075355.6 & $11.613 \pm 0.002$ & $11.022 \pm 0.002$ & $10.810 \pm 0.027$ & F6-G1 V & $0.35 \pm 0.02$ & $0.33_{-0.04}^{+0.05}$ \\
\hline $4 b$ & 185614.93 & +0753 30.7 & $14.950 \pm 0.005$ & $12.661 \pm 0.006$ & $11.483 \pm 0.004$ & G2-3 V & $2.19_{-0.02}^{+0.01}$ & $0.15_{-0.01}^{+0.16}$ \\
\hline $7 \mathrm{~b}$ & 185618.78 & +07 5430.9 & $13.445 \pm 0.005$ & $12.788 \pm 0.011$ & $12.508 \pm 0.009$ & G8-K0 V & $0.25_{-0.05}^{+0.03}$ & $5.44_{+1.30}^{-0.54}$ \\
\hline \multicolumn{9}{|c|}{ Giant stars: } \\
\hline $5 \mathrm{a}$ & 185611.00 & +075725.6 & $12.121 \pm 0.018$ & $10.265 \pm 0.013$ & $9.188 \pm 0.021$ & G4-7 III & $1.58 \pm 0.02$ & $0.57_{+0.04}^{-0.03}$ \\
\hline $7 \mathrm{a}$ & 185606.61 & +075712.0 & $17.490 \pm 0.036$ & $13.989 \pm 0.010$ & $12.225 \pm 0.005$ & K0-2 III & $3.02_{-0.08}^{+0.05}$ & $1.67_{+0.83}^{-0.32}$ \\
\hline $6 \mathrm{~b}$ & 185616.22 & +075446.8 & $17.764 \pm 0.051$ & $13.576 \pm 0.010$ & $11.606 \pm 0.004$ & M0-1 III & $3.39_{-0.05}^{-0.08}$ & $3.11_{+1.18}^{+0.83}$ \\
\hline
\end{tabular}

Notes. We include the equatorial coordinates, infrared magnitudes $\left(J, H, K_{\mathrm{S}}\right)$, and spectral type for each star. For those stars with estimated luminosity class, we have included the extinction and distance estimates.

the telluric correction spectrum, we see an absorption at the same position. We understand it to be an artifact from the reduction process and not a real stellar line. The Brackett series for star 2a is wider than the other star series, indicating that star 2 a could be a fast rotator.

In the case of spectrum $9 \mathrm{a}$, we also see an emission feature close to the HI (4-12) line, also seen in the 8a spectrum, that can be identified as an Fe II line. This line indicates the presence of circumstellar material, and can be found in Herbig Be stars (Puga et al. 2010). Because for star 9a we observe its Brackett series in absorption, we understand that this object is as very young, but already in the main sequence.

In the spectra of stars $12 \mathrm{a}$ and $1 \mathrm{~b}$ we observe an $H$ band spectrum similar to a B3 V star (Meyer et al. 1998), with a Brackett series extending until $\mathrm{HI}(4-15)$, and a clear He I at $1.70 \mu \mathrm{m}$. The $H$ band spectrum indicates a B3 V spectral type for both stars.

The Brackett series for spectrum 4a extends until H I (4-15) line and looks similar to that observed in B3 V stars (Meyer et al. 1998; Ranade et al. 2004). Around $1.70 \mu \mathrm{m}$ we find a weak feature, that could be a He I line, and the $K$ band spectrum is similar to the one observed in B3-5 V type stars (Hanson et al. 1996, 1998), which we adopt as spectral type for 4a.

Finally there are two stars in this group without a clear spectral classification. The first one is star 3a, with an irregular Brackett series and an asymmetric $\mathrm{Br} \gamma$ line. We see the He I lines at $1.70 \mu \mathrm{m}$ and at $2.06 \mu \mathrm{m}$, but the last one is located at a wavelength with a problematic telluric correction. Because this spectrum is noisy and most of its Brackett series is within the noise level, we cannot clearly classify it. Both the presence of He I at $1.70 \mu \mathrm{m}$ and the absence of He II limit the star 3a spectral type to early B-type.

The second star, $8 \mathrm{a}$, presents the Brackett series in strong emission for both $H$ and $K$ bands. No extended emission was detected during the reduction process, indicating that the emission comes from the immediate vicinity of the star. Its spectrum shows FeII at $1.64 \mu \mathrm{m}$ in emission, which indicates that it is very young, probably still surrounded by circumstellar material, as described by Puga et al. (2010) for their star 26. Because of the complete emission Brackett series, the emission Fe II line at $1.64 \mu \mathrm{m}$ and the colours of this star, we classify it as a young stellar object (YSO).

\subsubsection{A-, F-, and G-type dwarfs}

The observation of this type of stars was expected as contamination, because the pseudocolour $Q_{\mathrm{IR}}$ for OB and AF-type dwarfs is similar. The earliest star in this group, $8 \mathrm{~b}$, only presents in its spectrum a clear Brackett series, extended until the H I (4-18) line. No metallic lines are clearly distinguished in this spectrum, and we assign it the A5 III-V spectral type.

For F-type stars, the Brackett series begins to diminish, and $\mathrm{Mg}$ I lines at $1.57,1.71$, and $2.2 \mu \mathrm{m}$ become stronger. In the star 9b spectrum the HI (4-11), (4-12), and (4-13) lines are present and similar to those observed in F3 V (Meyer et al. 1998) or F7 V stars (Meyer et al. 1998). Even if Mg I is not clearly seen in the star $9 \mathrm{~b}$ spectrum, we assign the F3-7 V spectral type. 


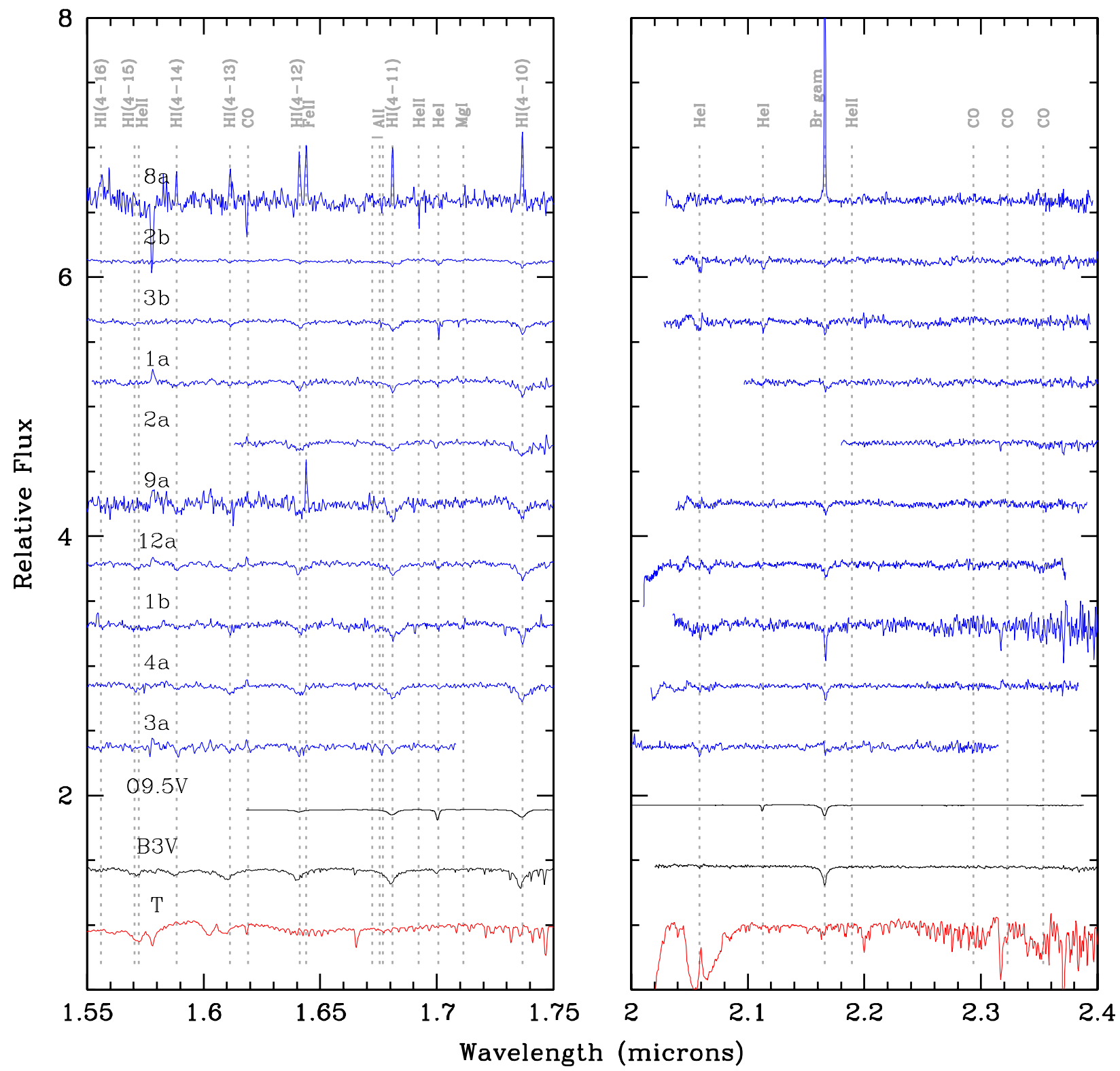

Fig. 7. Individual spectra in $H(l e f t)$ and $K$ band (right) for OB-type stars. Spectral features used in the spectral classification are labelled in grey. We show in black two spectra from the spectral libraries for comparison: O9.5 V HD 37468 (Hanson et al. 2005, degraded to our resolution) and B3 V HR5191 (Meyer et al. 1998). The telluric correction spectrum is shown in red.

For stars 6a (F6 V), 11a (F6-8 V), and 10a (F8 V), the Brackett series does not extend beyond the H I (4-13) line, their $\mathrm{Br} \gamma$ lines are narrow and deep, and the $\mathrm{Mg}$ I lines in the $H$ band spectrum are similar to those seen in late F V stars (Meyer et al. 1998).

The spectrum of star $5 \mathrm{~b}$ presents late F-type features and a faint ${ }^{12} \mathrm{CO}(2,0)$ band. The presence of this band indicates an early G-type for this star. The $H$ band spectrum is similar to an F6 V star (Meyer et al. 1998), but the $K$ band spectrum is more similar to an F8.5 V star, considering the Br $\gamma$ line's depth and the $\mathrm{Mg}$ I line at $2.28 \mu \mathrm{m}$ (Meyer et al. 1998). Because of the faint presence of the $\mathrm{CO}$ band, we assign this star a spectral type between F6 and G1 V.

The spectrum of star $4 \mathrm{~b}$ presents clear $\mathrm{MgI}$ lines in $H$ and $K$ bands; its $\mathrm{Mg}$ I lines at 1.57 and $1.71 \mu \mathrm{m}$ are broad but not as deep as expected for a $\mathrm{G} 2 \mathrm{~V}$ star. The $K$ band spectrum does not show the $\mathrm{Br} \gamma$ line, discarding a luminosity class III for this object, and its ${ }^{12} \mathrm{CO}(2,0)$ band fit the band from a G2-3 V star.
In the case of the star $7 \mathrm{~b}$, we observe that the $\mathrm{Mg}$ I lines are similar in depth and shape, to a $\mathrm{G} 8 \mathrm{~V}$ or $\mathrm{K} 0 \mathrm{~V}$ star. The $K$ band spectrum shows a shallow ${ }^{12} \mathrm{CO}(2,0)$ band and a deep $\mathrm{Ca}$ I line at $2.27 \mu \mathrm{m}$. Even if the deep of the $\mathrm{CO}$ band is similar to the $\mathrm{K} 0 \mathrm{~V}$ or $\mathrm{K} 5 \mathrm{~V} \mathrm{CO}$ band, the depth of the $\mathrm{CaI}$ line indicates an earlier spectral type for this object, between $\mathrm{G} 8$ and $\mathrm{K} 0 \mathrm{~V}$.

\subsubsection{Giant stars}

The three stars from this group present late-type spectral lines that allow us to classify them as luminosity class III. The spectra of stars $5 \mathrm{a}, 7 \mathrm{a}$, and $6 \mathrm{~b}$ have clear ${ }^{12} \mathrm{CO}(3,0)$ bands in $H$, characteristic of luminosity classes I or III, and not observed in stars with luminosity class V. The equivalent widths for the ${ }^{12} \mathrm{CO}(2,0)$ band in $K$, between $2.294 \mu \mathrm{m}$ and $2.304 \mu \mathrm{m}$, are $E W_{6 \mathrm{~b}}=17.60 \AA, E W_{5 a}=4.23 \AA$, and $E W_{7 a}=8.96 \AA$; these values fit better a luminosity class III for the three stars, 


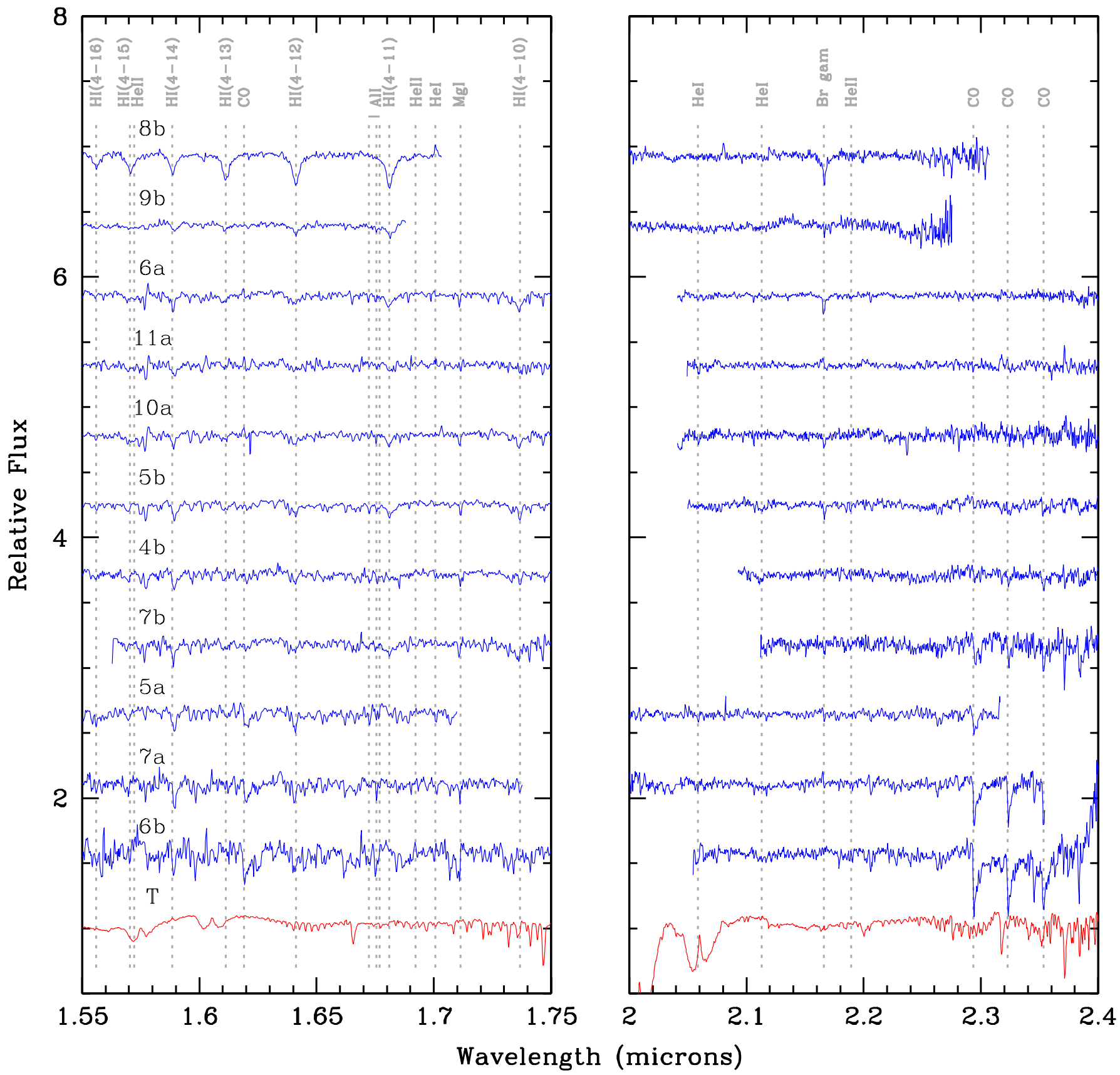

Fig. 8. Individual spectra in $H(l e f t)$ and $K$ band (right) for the AFG-type dwarfs and the giant stars. We show in grey the spectral lines used for the spectral classification. The red spectrum corresponds to the telluric correction spectrum, shown for spectral feature comparison.

according to the equivalent width and luminosity class relation given by Davies et al. (2007), and the spectral type adopted for these three stars.

The star 5a spectrum is similar to a G4III or a G7III spectra (Wallace \& Hinkle 1997) in the $K$ band. In the $H$ band it is similar to a G6 III (Meyer et al. 1998). We adopt the spectral type G4-7 III for this star.

The CO depth in $H$ for star $7 \mathrm{a}$ is similar to that observed in the HR 7949 spectrum (spectral type K0 III, Meyer et al. 1998), and the CO band in $K$ fits the HR 8694 (spectral type K0 III, Wallace \& Hinkle 1997) or the HR 6299 band (spectral type K2 III, Wallace \& Hinkle 1997). For this star we adopt a spectral type K0-2 III.

Finally, for the star $6 \mathrm{~b}$ we have adopted a M0-1 III spectral type, based on its $K$ band spectrum, which is similar to the HR 4069 and the HR 7635 spectra (spectral types M0 III, Wallace \& Hinkle 1997), and its $H$ band spectrum, similar to the HR 4517 spectrum (spectral type M1 III, Meyer et al. 1998).

\section{Discussion}

\subsection{Distance estimate}

From the stars observed spectroscopically, 19 out of 21 were selected following the pseudocolour $Q_{\mathrm{IR}}$ criteria (including star 11a, which has $Q_{\mathrm{IR}}=0.25$, slightly beyond the upper limit). The other two (i.e. $7 \mathrm{a}$ and $6 \mathrm{~b}$ ) were chosen because of their field position. From the 19 stars, 10 were classified as OB-type dwarfs. 
For all the stars but one (i.e. star 8a), we derive individual distances by comparing apparent and intrinsic magnitudes according to the assigned spectral type. We assume visual magnitudes from Cox (2000), intrinsic infrared colours from Tokunaga (2000), and the extinction law from Rieke et al. (1989) with $R=3.09$ (Rieke \& Lebofsky 1985). The choice of this extinction law was justified in Sect. 3.1. The $K_{\mathrm{S}}$-filter extinction, expressed as

$A_{K_{\mathrm{S}}}=\frac{E_{J-K_{\mathrm{S}}}}{1.514}$

varies between $0.04 \mathrm{mag}$ and $4.19 \mathrm{mag}$, equivalent to $A_{V}$ between 0.37 mag and 38.66 mag.

Distance errors were dominated by the spectral type uncertainties, which we estimate by deriving the individual distance for the same star assuming \pm 2 spectral subtypes. The distance errors associated with photometric errors were generally less than $10 \%$ of those derived from the spectral type uncertainty and therefore were not included in the error estimates. For stars with a range in spectral type (for example star 1a, B2-3 V), we assume the individual distance estimate to be the mean between the distance associated with each spectral type defining the range.

In Table 2 we present the individual extinction and distance determination for the OB-type stars used in the cluster distance estimate. These estimates are also shown in Fig. 9, where we present regions $\mathrm{A}$ (red) and $\mathrm{B}$ (blue) individual distances and extinctions for OB (left) and non-OB (right) type stars. In the figure we show the mean distance value for regions A (threepointed star) and B (four-pointed star), and we can observe that:

- In region $\mathrm{A}$, OB-type stars have a mean distance of $1.87_{-0.77}^{+1.29} \mathrm{kpc}$. For this determination, we have excluded stars $8 \mathrm{a}$ and $9 \mathrm{a}$, both belonging to the nucleus of region $\mathrm{A}$. Because star 8a has not been spectroscopically classified, the extinction and distance could not be estimated for this object. For star 9a, the spectroscopically observed star with the largest reddening, photometry is probably contaminated by local nebulosity, making the measured magnitude unreliable. Even without an extinction or distance estimate for stars 8a and 9a, their positions in the CCDs indicate that these stars are not foreground objects.

- In region B, a mean distance of $1.95_{-0.76}^{+1.21} \mathrm{kpc}$ was derived from stars $1 b, 2 b$, and, $3 b$ individual distances. This distance is in agreement within errors with the one derived for IRAS 18537+0753: $2.1 \mathrm{kpc}$ (Plume et al. 1992), and the estimate derived from the observations of the methanol maser [HHG86] 185345.9+074916: $2.2 \mathrm{kpc}$ (Val'tts et al. 2000). We conclude that stars $1 \mathrm{~b}, 2 \mathrm{~b}$, and $3 \mathrm{~b}$ are associated with the IRAS source IRAS 18537+0753, the methanol maser, and the massive star forming region Sh2-76 E.

- Dwarfs stars with spectral type A, F, or G have individual distances less than $1 \mathrm{kpc}$. Consequently we classify these objects as foreground dwarf stars from the galactic disc. The exception in this group is star $7 \mathrm{~b}$, with a distance estimate of $d=5.44 \mathrm{kpc}$. After reviewing the LIRIS images we notice that this star is contaminated by a close companion, and their radial profiles are merged. Because of this contamination, we exclude the star $7 \mathrm{~b}$ from the analysis.

- Giant stars have two types of extinction and distance values: the foreground star 5a, probably located in the same structure as A-, F-, and G-type stars; and the background giant stars $5 \mathrm{a}$ and $6 \mathrm{~b}$. In both cases these stars are part of the giant population of the galactic disc.

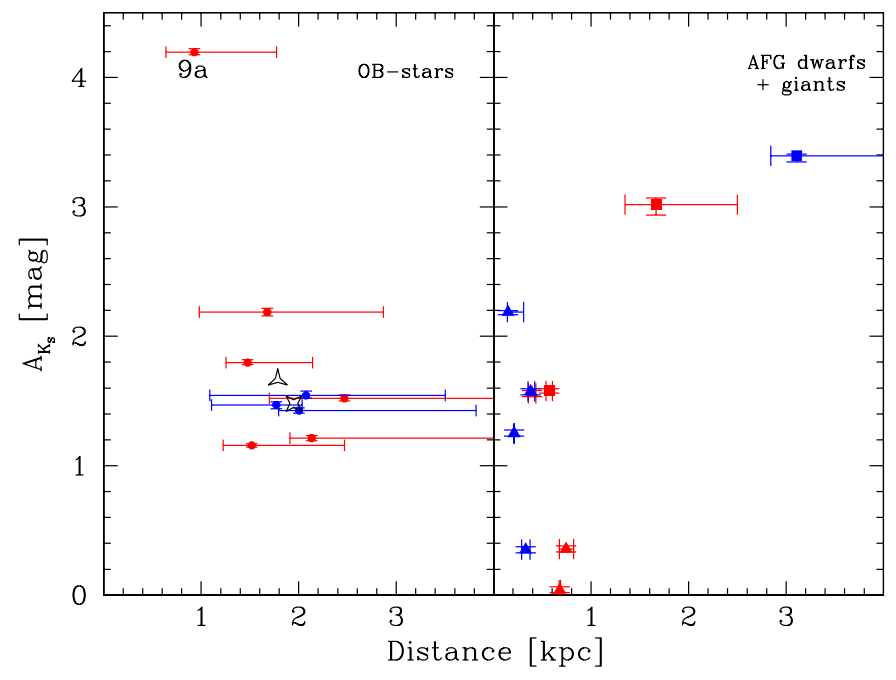

Fig. 9. Left: individual distances and extinctions for OB-type stars. Red symbols show the parameters for region A stars, and blue symbols for region B stars. Mean values of distance and extinction for regions A and B stars are marked with three- and four-pointed black stars, respectively. Right: individual distances and extinctions for non-OB stars in regions A (red symbols) and B (blue symbols). Estimates for spectral type $\mathrm{A}, \mathrm{F}$, and $\mathrm{G}$ dwarfs are shown with triangles, and giant stars estimates are shown with squares.

The OB-type stars from the cores in regions A and B are located at the same distance within errors. We thus conclude that they belong to two near-IR nuclei of a single, young, massive, and embedded stellar population. Using the mean distance of regions $\mathrm{A}$ and $\mathrm{B}$, we estimate a distance of $1.90_{-0.90}^{+1.28} \mathrm{kpc}$ for the Masgomas- 4 association, with a mean extinction $A_{K}=$ $1.54 \pm 0.02 \mathrm{mag}$. At this distance, the projected separation of regions $\mathrm{A}$ and $\mathrm{B}$ corresponds to a linear separation of about $2.4 \mathrm{pc}$.

In the CCDs (Fig. 5) we observe objects in the T Tauri region, below the black segmented line. The $\mathrm{T}$ Tauri candidates are present in both regions $\mathrm{A}$ and $\mathrm{B}$, but only in the case of region $\mathrm{B}$ we identify a group of objects with extra reddening (colours $\left(H-K_{\mathrm{S}}\right)>2$, shown with red circles in the CCD of region B). These stars (or protostars) are concentrated around the region $\mathrm{B}$ nucleus, as can be seen in Fig. 2, and their extra reddening can be explained by the presence of circumstellar accretion discs.

Because of the extra reddening of this group of stars, compared with the colours of the T Tauri objects, we classify them as Herbig Ae/Be candidates. According to Subramaniam et al. (2006), Herbig Ae/Be objects are located to the right of the locus of the classic T Tauri objects in the CCDs. These objects are also younger, more embedded, and more massive than $\mathrm{T}$ Tauri objects.

Like Masgomas-1 (Ramírez Alegría et al. 2012), Masgomas-4 is located in the direction of the RSG clusters. Our distance estimate discards a physical link of Masgomas-4 with the intersection between the close end of the Galactic bar and the Scutum-Centaurus arm base. The $1.90 \mathrm{kpc}$ distance estimate places Masgomas-4 closer than the Scutum-Centaurus arm, probably in the Carina-Sagittarius arm.

\subsection{Mass and age estimates}

To estimate the cluster's present-day total mass, we follow a similar procedure to that described by Ramírez Alegría et al. (2012). We fit a Kroupa (Kroupa 2001) initial mass function (IMF) to the cluster present-day mass function, and integrate it 


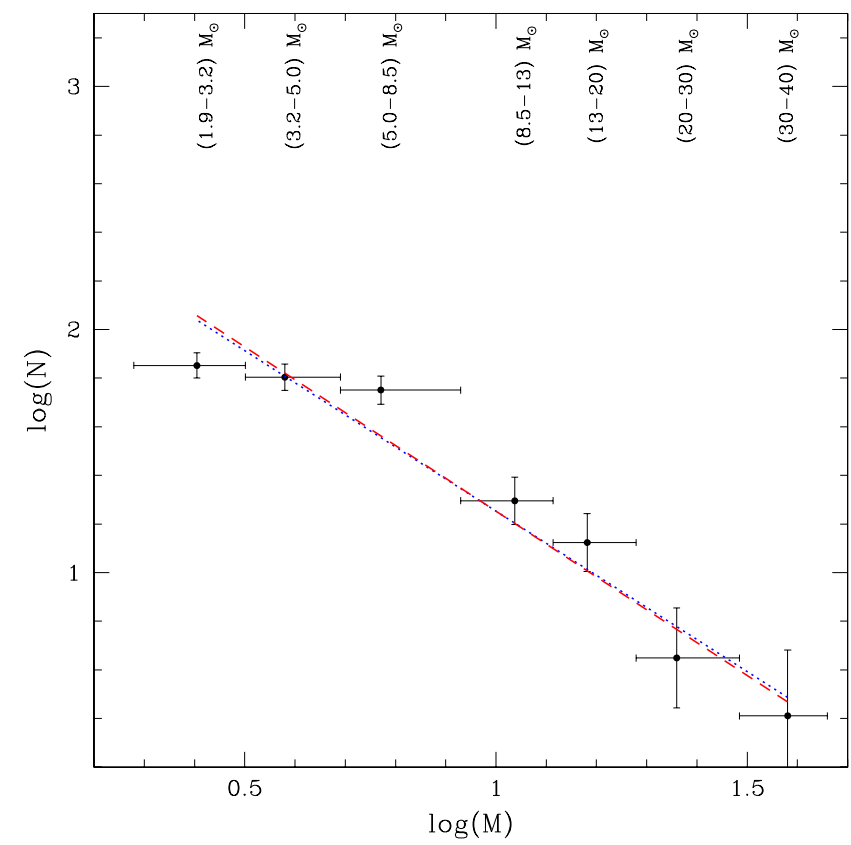

Fig. 10. Present-day mass function for Masgomas-4. Points show the central position in the mass ranges indicated above them, and the segmented red line corresponds to the Kroupa IMF fitted to the data. As a comparison, we show the least-squares fitting (one degree function) to our data with a blue dotted line.

from $\log (M)=-1.0$ dex to 1.5 dex. Because there is not an evolved stellar population in Masgomas-4, we can assume that the difference between the initial and present mass function in the cluster is small.

We fit the Kroupa IMF to the cluster mass function between $\sim 2.5 M_{\odot}$ and $\sim 35 M_{\odot}$. The mass function was derived from the luminosity function and the contribution from the galactic disc stellar population was corrected using the observed control field (from LIRIS images). The cluster and control field photometries were cut in $J<16.5 \mathrm{mag}, H<15.5 \mathrm{mag}$, and $K<14.5 \mathrm{mag}$, to assure a completeness close to 1.0 , following the same procedure described by Ramírez Alegría et al. (2012).

To obtain the luminosity and mass functions, we project every star, following the reddening vector, to the main sequence located at $1.9 \mathrm{kpc}$. This sequence is defined by the colours and magnitudes given by Cox (2000), and is expressed analytically by two lines: one from the spectral type $09 \mathrm{~V}$ to $\mathrm{A} 0 \mathrm{~V}$, and the second one from $\mathrm{A} 0 \mathrm{~V}$ to $\mathrm{G} 0 \mathrm{~V}$, corresponding to the last spectral type to the limit magnitude chosen for completeness.

To derive the mass function we convert the $K_{\mathrm{S}}$ magnitudes to solar masses, using the values given by Cox (2000) for stars later than B0 V, and Martins et al. (2005) for stars earlier than this. For magnitudes that were between values in the catalogue, we interpolate between the two closest values.

After subtracting the Masgomas-4 field and the control field mass function, we obtain the cluster present-day mass function, shown in Fig. 10. We fit a Kroupa IMF and integrate in the mass range $0.10-31 M_{\odot}$ (equivalent to $\log (M)=-1.0 \mathrm{dex}$ to $1.5 \mathrm{dex}$ ), estimating a total cluster mass (lower limit) of $(2.19 \pm 0.31) \times$ $10^{3} M_{\odot}$. The Kroupa IMF fitting is also compared in the figure with a least-squares fitting to the data. Both fits are similar within data errors.

Cluster age can be limited by the presence of an $09 \mathrm{~V}$ star. This gives an upper limit of $10 \mathrm{Myr}$ that could be reduced by the confirmation of earlier stars in the centre of region $\mathrm{A}$, for example star 8a. This age limit of $10 \mathrm{Myr}$ is also dependent on the rotation speed assumed by the stellar evolutionary models (Brott et al. 2011).

The presence of $\mathrm{T}$ Tauri and Herbig Ae/Be candidates also gives information about the cluster age. According to Hernández et al. (2007) in young clusters (age $3 \mathrm{Myr}$ ), $30 \%$ of the T Tauri objects and $15 \%$ of the Herbig Ae/Be objects lose their circumstellar discs. The survivor discs around Herbig Ae/Be objects will be lost in less than $10 \mathrm{Myr}$.

Although there is a difference because of the presence of Herbig Ae/Be candidates in region $\mathrm{B}$, which are absent in region A, both cores display signs of on-going star formation (IRAS sources and masers). The presence of Herbig Ae/Be candidates also limits the age of the recent burst in the region $\mathrm{B}$ core to 5 Myr.

Lada \& Lada (2003) indicate that it is very unlikely to find embedded clusters older than 5 Myr. Because part of the stellar population of Masgomas-4 is still deeply embedded and the whole cluster is surrounded by an H II cloud, we adopt this value as a new upper limit for the cluster age, limiting the cluster age to $5 \mathrm{Myr}$.

\section{Conclusions}

We present the observations, physical estimates, and analysis for the young, double-core, and obscured cluster Masgomas-4. The near-IR observations, individual extinctions, and distance estimates for the OB-type star population are in agreement with a single association, with two active regions of stellar formation. The individual distances estimated for regions A and B point to a single distance of $1.90_{-0.90}^{+1.28} \mathrm{kpc}$, and this distance indicates that the cluster is associated with the IRAS source IRAS $18537+0753$, the methanol maser [HHG86] 185345.9+074916, and the massive star forming region Sh2-76 E. The Algol variable star V 1665 Aql is discarded as part of the Masgomas-4 stellar population.

We observe in both regions $\mathrm{A}$ and $\mathrm{B}$ massive stars, and spectroscopic evidence of young or forming stars, like central stars $8 \mathrm{a}$ (emission Brackett series and Fe II at $1.64 \mu \mathrm{m}$ in emission) and 9a (Fe II at $1.64 \mu \mathrm{m}$ in emission). Regions A and B present $\mathrm{T}$ Tauri candidates, according to their CCDs. In the case of region $\mathrm{B}$, we also detect a clearly concentrated population of Herbig Ae/Be candidates around Sh2-76E. Its exclusive presence around the region B core could indicate a different star formation history between regions A and B (due to initial mass or age) and a slightly younger population in the region B core.

The mass estimate, derived from integrating the Kroupa initial mass function fitted to our data, gives a lower limit for the cluster total mass of $(2.19 \pm 0.31) \times 10^{3} M_{\odot}$. The presence of $\mathrm{T}$ Tauri and Herbig Ae/Be candidates and the surrounding gas observed in mid-infrared images imply that Masgomas-4 is still a young and active object in terms of star formation. The very young obscured population and the surrounding gas allow us to limit the cluster age to less than 5 Myr.

Acknowledgements. S.R.A. was supported by the GEMINI-CONICYT project number 32110005, the FONDECYT project No. 3140605, and the investigation project Massive Stars in Galactic Obscured Massive Clusters. Part of this work was supported by the Science and Technology Ministry of the Kingdom of Spain (grants AYA2010-21697-C05-04 and AYA2012-39364-C02-01), the Gobierno de Canarias (PID2010119), and by the Chilean Ministry for the Economy, Development, and Tourism's Programa Iniciativa Científica Milenio through grant IC 12009, awarded to The Millennium Institute of Astrophysics (MAS). The William Herschel Telescope is operated on the island of La Palma by the Isaac Newton Group in the Spanish Observatorio del Roque de los Muchachos of the Instituto de Astrofísica de Canarias. This publication makes 
use of data products from the Two Micron All Sky Survey, which is a joint project of the University of Massachusetts and the Infrared Processing and Analysis Center/California Institute of Technology, funded by the National Aeronautics and Space Administration and the National Science Foundation. Average asymmetrical errors were estimated using the Java applet available at http://www . slac. stanford. edu/ barlow/statistics.html (Barlow 2004).

\section{References}

Alexander, M. J., Kobulnicky, H. A., Clemens, D. P., et al. 2009, AJ, 137, 4824 Barlow, R. 2004, unpublished [arXiv: physics/0406120]

Baudry, A., Desmurs, J. F., Wilson, T. L., \& Cohen, R. J. 1997, A\&A, 325, 255 Benjamin, R. A., Churchwell, E., Babler, B. L., et al. 2003, PASP, 115, 953

Bica, E., Dutra, C. M., \& Barbuy, B. 2003a, A\&A, 397, 177

Bica, E., Dutra, C. M., Soares, J., \& Barbuy, B. 2003b, A\&A, 404, 223

Borissova, J., Bonatto, C., Kurtev, R., et al. 2011, A\&A, 532, A131

Brott, I., de Mink, S. E., Cantiello, M., et al. 2011, A\&A, 530, A115

Chan, S. J., Henning, T., \& Schreyer, K. 1995, VizieR Online Data Catalog, $\mathrm{J} / \mathrm{A}+\mathrm{AS} / 115 / 285$

Chan, S. J., Henning, T., \& Schreyer, K. 1996, A\&AS, 115, 285

Clark, J. S., Negueruela, I., Davies, B., et al. 2009, A\&A, 498, 109

Comerón, F., \& Pasquali, A. 2005, A\&A, 430, 541

Cox, A. N. 2000, Allen's Astrophysical Quantities, 4th edn. (New York: Springer)

Davies, B., Figer, D. F., Kudritzki, R.-P., et al. 2007, ApJ, 671, 781

Davies, B., Bastian, N., Gieles, M., et al. 2011, MNRAS, 411, 1386

Dutra, C. M., Bica, E., Soares, J., \& Barbuy, B. 2003, A\&A, 400, 533

Eikenberry, S., Elston, R., Raines, S. N., et al. 2006, in Ground-based and Airborne Instrumentation for Astronomy, eds. I. S. McLean, \& M. Iye (Washington: SPIE), Proc. SPIE, 6269, 626917

Figer, D. F., MacKenty, J. W., Robberto, M., et al. 2006, ApJ, 643, 1166 Forbes, D. 1989, A\&AS, 77, 439

Fragoso-López, A. B., Acosta-Pulido, J. A., Hernández, E., et al. 2008, in Ground-based and Airborne Instrumentation for Astronomy II, eds.

I. S. McLean, \& M. M. Casali (Washington: SPIE), Proc. SPIE, 7014, 701468 Froebrich, D., Scholz, A., \& Raftery, C. L. 2007, MNRAS, 374, 399 González-Fernández, C., \& Negueruela, I. 2012, A\&A, 539, A100

Hanson, M. M., \& Popescu, B. 2008, in IAU Symp. 250, eds. F. Bresolin, P. A. Crowther, \& J. Puls, 307

Hanson, M. M., Conti, P. S., \& Rieke, M. J. 1996, ApJS, 107, 281

Hanson, M. M., Rieke, G. H., \& Luhman, K. L. 1998, AJ, 116, 1915

Hanson, M. M., Kudritzki, R., Kenworthy, M. A., Puls, J., \& Tokunaga, A. T. 2005, ApJS, 161, 154

Hanson, M. M., Kurtev, R., Borissova, J., et al. 2010, A\&A, 516, A35

Hernández, J., Hartmann, L., Megeath, T., et al. 2007, ApJ, 662, 1067
Ibanoğlu, C., Evren, S., Taş, G., et al. 2007, MNRAS, 380, 1422

Kroupa, P. 2001, MNRAS, 322, 231

Kurtev, R., Borissova, J., Georgiev, L., Ortolani, S., \& Ivanov, V. D. 2007, A\&A, 475, 209

Kurtev, R., Ivanov, V. D., Borissova, J., \& Ortolani, S. 2008, A\&A, 489, 583

Lada, C. J., \& Lada, E. A. 2003, ARA\&A, 41, 57

Lawrence, A., Warren, S. J., Almaini, O., et al. 2007, MNRAS, 379, 1599

Manchado, A., Barreto, M., Acosta-Pulido, J., et al. 2004, in Ground-based and Airborne Instrumentation for Astronomy, eds. A. F. M. Moorwood, \& M. Iye (Washington: SPIE), Proc. SPIE, 5492, 549188

Marín-Franch, A., Herrero, A., Lenorzer, A., et al. 2009, A\&A, 502, 559

Martins, F., Schaerer, D., \& Hillier, D. J. 2005, A\&A, 436, 1049

Mercer, E. P., Clemens, D. P., Meade, M. R., et al. 2005, ApJ, 635, 560

Meyer, M. R., Edwards, S., Hinkle, K. H., \& Strom, S. E. 1998, ApJ, 508, 397

Minniti, D., Lucas, P. W., Emerson, J. P., et al. 2010, New Astron., 15, 433

Negueruela, I., \& Schurch, M. P. E. 2007, A\&A, 461, 631

Negueruela, I., González-Fernández, C., Marco, A., Clark, J. S., \& Martínez-Núñez, S. 2010, A\&A, 513, A74

Negueruela, I., González-Fernández, C., Marco, A., \& Clark, J. S. 2011, A\&A, 528, A59

Newberry, M. V. 1991, PASP, 103, 122

Palagi, F., Cesaroni, R., Comoretto, G., Felli, M., \& Natale, V. 1993, A\&AS, 101,153

Plume, R., Jaffe, D. T., \& Evans, II, N. J. 1992, ApJS, 78, 505

Puga, E., Marín-Franch, A., Najarro, F., et al. 2010, A\&A, 517, A2

Ramírez Alegría, S., Herrero, A., Marín-Franch, A., et al. 2011, A\&A, 535, A8

Ramírez Alegría, S., Marín-Franch, A., \& Herrero, A. 2012, A\&A, 541, A75

Ranade, A., Gupta, R., Ashok, N. M., \& Singh, H. P. 2004, Bull. Astron. Soc. India, 32, 311

Rieke, G. H., \& Lebofsky, M. J. 1985, ApJ, 288, 618

Rieke, G. H., Rieke, M. J., \& Paul, A. E. 1989, ApJ, 336, 752

Saito, R. K., Hempel, M., Minniti, D., et al. 2012, A\&A, 537, A107

Sharpless, S. 1959, ApJS, 4, 257

Skrutskie, M. F., Cutri, R. M., Stiening, R., et al. 2006, AJ, 131, 1163

Slysh, V. I., Val'tts, I. E., Kalenskii, S. V., et al. 1999, A\&AS, 134, 115

Stetson, P. B. 1994, PASP, 106, 250

Subramaniam, A., Mathew, B., Bhatt, B. C., \& Ramya, S. 2006, MNRAS, 370, 743

Szymczak, M., Hrynek, G., \& Kus, A. J. 2000, A\&AS, 143, 269

Tokunaga, A. T. 2000, in Infrared Astronomy (Springer), 143

Vacca, W. D., Cushing, M. C., \& Rayner, J. T. 2003, PASP, 115, 389

Val'tts, I. E., Ellingsen, S. P., Slysh, V. I., et al. 2000, MNRAS, 317, 315 Wallace, L., \& Hinkle, K. 1997, ApJS, 111, 445

Zinchenko, I., Forsstroem, V., Lapinov, A., \& Mattila, K. 1994, A\&A, 288, 601

Zinchenko, I., Henning, T., \& Schreyer, K. 1997, A\&AS, 124, 385 